Juntendo Medical Journal

2016. 62 (Suppl 1), 124

\title{
Long Term Effect of Cardiorespiratory Fitness for a Prevention Against Diabetes
}

\author{
YUKI SOMEYA*1) 2), SACHIO KAWAI*3), Yoshimitsu KOHMURA*2), \\ KAZUHIRO AOKI*2), HIROYUKI DAIDA*4)
}

*1) Sportology Center, Juntendo University Graduate School of Medicine, Tokyo, Japan, *2) Faculty of Health and Sports Science, Juntendo University, Chiba, Japan, ${ }^{* 3}$ Graduate School of Health and Sports Science, Juntendo University, Chiba, Japan, *4) Department of Cardiovascular Medicine, Juntendo University Graduate School of Medicine, Tokyo, Japan

In Japan, although the prevalence of overweight $(\mathrm{BMI} \geq 25)$, the strongest predisposing factor for type 2 diabetes, is low compared with that in Europe and the United States, the prevalence of type 2 diabetes has increased over the last 15 years. In epidemiological studies of both Japanese and Caucasian population, a high level of cardiorespiratory fitness has been shown to be a protective factor against type 2 diabetes. However, there are no reports focused specifically on athletes that investigate whether high cardiorespiratory fitness at a young age can prevent disease later in life. Therefore, the present study examined the relationship between cardiorespiratory fitness at a young age and the development of type 2 diabetes in Japanese male college athletes using a cohort study.

Between 2007 and 2009, 3,539 male alumni who graduated at the physical education department, were mailed a follow-up questionnaire about their diabetes background and 1,385 male alumni returned it. The cardiorespiratory fitness of male alumni, as measured by 1,500-m endurance run in college (1971-1991) was available for 41\% of the respondents. In the present study, we analyzed for 570 male alumni by Cox's proportional hazards models and adjusted for age, year of graduation, BMI, smoking, and sports club participation at college age.

This study covered a 26-year follow-up period (Interquartile Range: IQR: 23-29 years), and median age at the follow-up questionnaire was 49 years (IQR: 45-52 years). At follow-up, 22 men had developed type 2 diabetes. The incidence of type 2 diabetes was inversely correlated with cardiorespiratory fitness, and the low cardiorespiratory fitness level increased the cumulative incidence rate of type 2 diabetes. There were progressively lower age-adjusted relative risks of type 2 diabetes across cardiorespiratory fitness levels $(p=0.01$ for trend). After adjustment for age, year of graduation, BMI, smoking, and sports participation, hazards ratio and 95\% CI by category (low, medium, and high) were 1.00 (reference), $0.40(0.14-1.13)$ and $0.26(0.07-1.00)(\mathrm{p}=$ 0.03 for trend).

We conclude that cardiorespiratory fitness at a young age can predict type 2 diabetes later in life among Japanese male athletes.

* This data was already published at BMC Public Health. And this report was extracted from Someya Y, et al: Cardiorespiratory fitness and the incidence of type 2 diabetes: a cohort study of Japanese male athletes. BMC Public Health, 2014; 14: 493.

Key words: cardiorespiratory fitness, diabetes, young age, Japanese, athlete

Corresponding author: Yuki Someya Sportology Center, Juntendo University Graduate School of Medicine 2-1-1 Hongo, Bunkyo-ku, Tokyo 113-8421, Japan

TEL: +81-3-5802-1759 FAX: +81-3-3813-5996 E-mail: yksomeya@juntendo.ac.jp

The $2^{\text {nd }}$ Congress, International Academy of Sportology 〔Held on Sep. 12, 2015〕

〔Received Dec. 18, 2015〕 